\title{
Diferencias morfométricas de Gambusia holbrooki (Pisce: Poeciliidae) que habitan en los estanques de regadío de los valles de Lluta y Azapa, Chile
}

\author{
Differences morphometric Gambusia holbrooki (Pisce: Poeciliidae) \\ inhabiting in irrigation ponds of valleys of Lluta and Azapa, Chile \\ José López-Cepeda ${ }^{1 *}$, Patricia Pacheco-Cartagena ${ }^{2}$, Arnaldo Vilaxa-Olcay ${ }^{1,3}$
}

\begin{abstract}
RESUMEN
En los estanques de regadío ubicados en los valles de Lluta y Azapa habita el pez llamado Gambusia holbrooki. Este pez fue introducido en los sistemas límnicos de la Región de Arica y Parinacota en 1937 y se ha adaptado a las condiciones abióticas de las aguas que se suministran a los estanques de regadío. La investigación tiene como objetivo determinar las diferencias morfométricas de dos poblaciones de gambusias en relación con las características del agua del valle de Lluta y Azapa, en particular a la concentración de boro. En enero de 2013 se capturaron 457 peces adultos en ambos valles para observar cambios morfométricos, medir los niveles de boro presente en el hábitat y en los tejidos de G. holbrooki. Los resultados demuestran que existen cambios morfométricos entre las dos poblaciones, los peces de Lluta son más grandes que los peces de Azapa, mientras que el contenido de boro en los tejidos no varía significativamente $(\mathrm{p}=0,07)$, en ambas poblaciones.
\end{abstract}

Palabras clave: estanque de regadío, Gambusia holbrooki, boro, microhábitat, zona árida.

\begin{abstract}
In the ponds for irrigation found in the Lluta and Azapa valleys, inhabit a fish called Gambusia holbrooki. This fish was introduced in the Arica and Parinacota Region limnic systems in 1937 and it has adapted to abiotic conditions of waters supplying irrigation ponds. The investigation aims to determine the differences morphometric of two populations of mosquitofish in relation to the characteristics of the water of the valley of Lluta an Azapa, in particular to the concentration of boron. 457 fish were captured in January 2013 adults in both valleys to observe morphometric changes, as they were also measured levels of boron in the habitat and in the tissues of $\mathrm{G}$. holbrooki. The results show that there are morphometric changes between the two populations, Lluta fish are larger than the fish of Azapa, while the content of boron in the tissues does not vary significantly $(p=0.07)$, in both populations.

Key words: pond for irrigation, Gambusia holbrooki, boron, microhabitat, arid zone.
\end{abstract}

\section{Introducción}

Los agricultores del valle de Lluta y Azapa construyen estanques dentro de sus predios para acumular agua para sus actividades agrícolas, en el valle de Lluta el agua que se acumula es del propio río, mientras que en el valle de Azapa las fuentes de agua son dos, aguas superficiales y subterráneas, que provienen del río San José, y agua trasvasada desde la cuenca del río Lauca, siendo la principal fuente de agua para la agricultura del valle de Azapa. Por lo que las características del agua del estanque en ambos valles difieren. Las aguas que provienen del río Lluta tienen una alta salinidad, con una conductividad eléctrica sobre $2 \mathrm{ds} / \mathrm{m}$ y concentraciones de boro superior a $11 \mathrm{ppm}$, en cambio la conductividad eléctrica del agua superficial de la cuenca del río San José y la trasvasada desde la cuenca del río Lauca es menor a $1 \mathrm{ds} / \mathrm{m}$, mientras que el boro se encuentra

\footnotetext{
1 Departamento de Biología, Facultad de Ciencias, Universidad de Tarapacá, Avda. General Velásquez 1775, Arica, Chile.

2 Laboratorio de Análisis de Suelos y Agua, Facultad de Ciencias Agronómicas, Universidad de Tarapacá, Avda. General Velásquez 1775, Arica, Chile.

3 Centro de Ciencias Marinas y Limnológicas, Facultad de Ciencias, Universidad de Tarapacá, Avda. General Velásquez 1775, Arica, Chile.

* Autor para correspondencia: jlopez@uta.cl
}

Fecha de Recepción: 7 Noviembre, 2013.

Fecha de Aceptación: 15 Junio, 2014. 
en concentraciones inferiores a $1 \mathrm{ppm}$ (Torres y Acevedo, 2008), algunos estudios en ciencias agrícolas que abordan los factores abióticos que afectan a cultivos de cada valle los han realizado Osorio et al. (2013) y Figueroa et al. (1994), sin prestar atención a los microhábitat que se generan en los estanques de regadío.

Actualmente, en ambos ríos, se ha identificado a la especie Gambusia holbrooki como el pez predominante (UTA, 1997; CADE-IDEP, 2004; PUC, 2009). El éxito en la colonización de los sistemas acuáticos de este pez se debió a las características climáticas de la Región de Arica y Parinacota que favorece la sobrevivencia de $G$. holbrooki, por otro lado también es un ambiente ideal para la proliferación del mosquito Anopheles pseudopunctipennis portador de la malaria, por lo que en 1937 se adoptaron medidas para erradicar al estado acuático-larval del insecto (por ende la malaria), lo que incluyó la diseminación de Gambusia holbrooki en los ríos Lluta y San José (Neghme et al., 1947). Por las características predatorias del pez, este fue utilizado como agente biológico controlador de poblaciones de mosquito, no tan solo en Chile sino que en gran parte del mundo, por tal motivo G. holbrooki está prácticamente presente en todos los sistemas límnicos del mundo (Pyke, 2005; Oriol et al., 2012), de hecho se considera como el pez dulceacuícola de mayor abundancia y con rangos de distribución más amplios del planeta, por su plasticidad biológica, capaz de soportar condiciones ambientales altamente estresantes, con registros de poblaciones de $G$. holbrooki en ambientes salinos de $45 \mathrm{~g} / \mathrm{l}$ (Pyke, 2005; Ruiz-Navarro et al., 2011).

Gambusia holbrooki no es una especie nativa, aunque está considerado en los informes técnicos que se presentan para caracterizar la biota acuática de la Región de Arica y Parinacota como parte de la fauna local (UTA, 1997; PUC, 2009), se desconoce el impacto ecológico o las adaptaciones biológicas que ha tenido que modificar este pez para colonizar los ríos de la Región. Estudios indican que Paralichthys olivaceus y Pagrus major aumentan su tamaño corporal en presencia de boro y como también Gambusia puncticulata frente a la contaminación antrópica (Furuta et al., 2007; Cabrera et al., 2008). Este estudio pretende dar cuenta de los cambios merísticos en dos poblaciones de G. holbrooki que habitan en estanques de regadío de Lluta y Azapa en atención a la calidad del agua.

\section{Material y Métodos}

\section{Obtención de muestras}

Para este estudio se recolectaron 457 peces adultos, fueron capturados en estanques de regadío de los valles de Lluta (18²4'40,4" S-7007'18,16”' O)

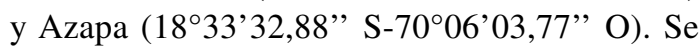
obtuvo una muestra de agua de cada estanque de regadío junto con los peces capturados. Las muestras de agua y material biológico fueron enviadas al Laboratorio de Suelo y Agua de la Universidad de Tarapacá para su análisis por medio de espectrofotometría con énfasis en la presencia de boro, para observar cuánta absorción de este elemento hay en los tejidos del pez, dependiendo del lugar que habite.

\section{Caracterización merística de ambas poblaciones y análisis estadísticos}

Los peces capturados fueron medidos con un pie de metro con una sensibilidad de $\pm 0,005$ milímetro (mm) y pesados en una balanza analítica a $\pm 0,00005$ milígramo (mg) de sensibilidad y se obtuvieron los siguientes datos: longitud total $(\mathrm{Lt})$, que corresponde el largo medido de boca a cola; peso total (Pt), que corresponde al peso húmedo del cuerpo completo del pez. En todos los peces se calculó el factor de condición (K) de la siguiente forma:

$$
K=\frac{P t}{L t^{3}} * 100
$$

Lo que busca el factor de condición es saber cuánta masa corporal puede incorporar el pez con el alimento disponible, interpretándose como el "bienestar" de un pez o población, basándose en que los peces de mayor peso y tamaño presentan una mejor condición (Granado, 2002; Cifuentes et al., 2012).

Para el análisis estadístico se utilizó el test de Fisher para identificar la normalidad de los datos y a posterior se utilizó el test de Student para dos muestras suponiendo varianzas iguales o desiguales con nivel de significancia de $\alpha=0,05$. 


\section{Resultados y Discusión}

\section{Descripción morfométrica de Gambusia holbrooki capturados en los valles de Lluta y Azapa}

Los resultados de los t- test realizados para observar diferencia de tamaños entre machos y hembras, como los valores de peso promedio agrupados por lugar de origen, se muestran en la Tabla 1. Los datos obtenidos para caracterizar morfológicamente los ejemplares capturados en ambos ríos demuestran que, independiente del lugar de origen, el marcado dimorfismo sexual que ostenta Gambusia holbrooki se mantiene inalterable, es decir, las hembras son más grandes que los machos (Figura 1), lo que coincide con lo informado por Pyke (2005) en una revisión que hace a la biología de G. holbrooki; de igual forma nuestro estudio concuerda con los tamaños máximos que puede alcanzar esta especie (Ruiz-Navarro et al., 2011).

Al observar el factor de condición que obtuvieron las dos poblaciones (Lluta y Azapa), es decir, cómo se comporta la relación peso/tamaño de los peces,

Tabla 1. Contiene los valores medios \pm desviación estándar para longitud total $(\mathrm{mm})$, peso total (mg) y factor de condición; como a su vez la probabilidad asociada al test de Student con $\alpha=0,05$, de peces hembras y machos que habitan los valles de Lluta (río Lluta) y Azapa (río San José), enero 2013.

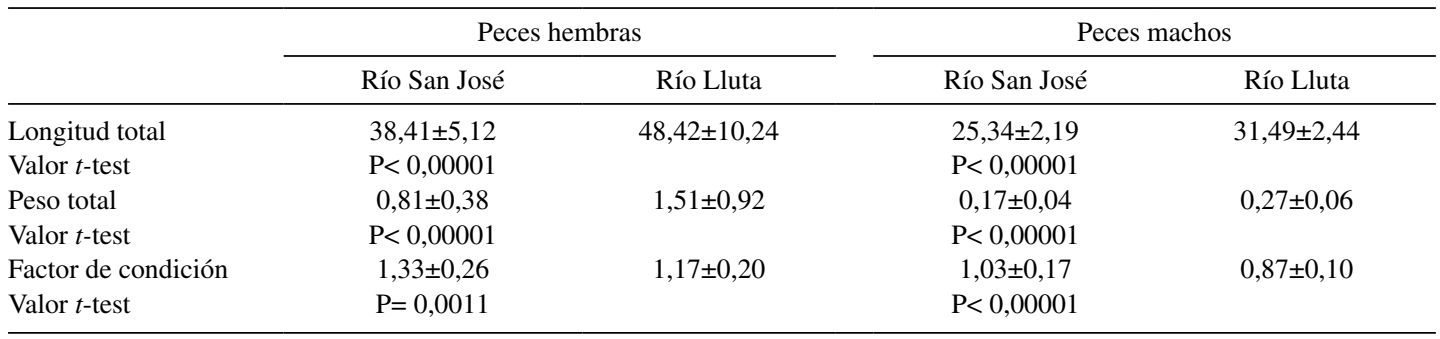

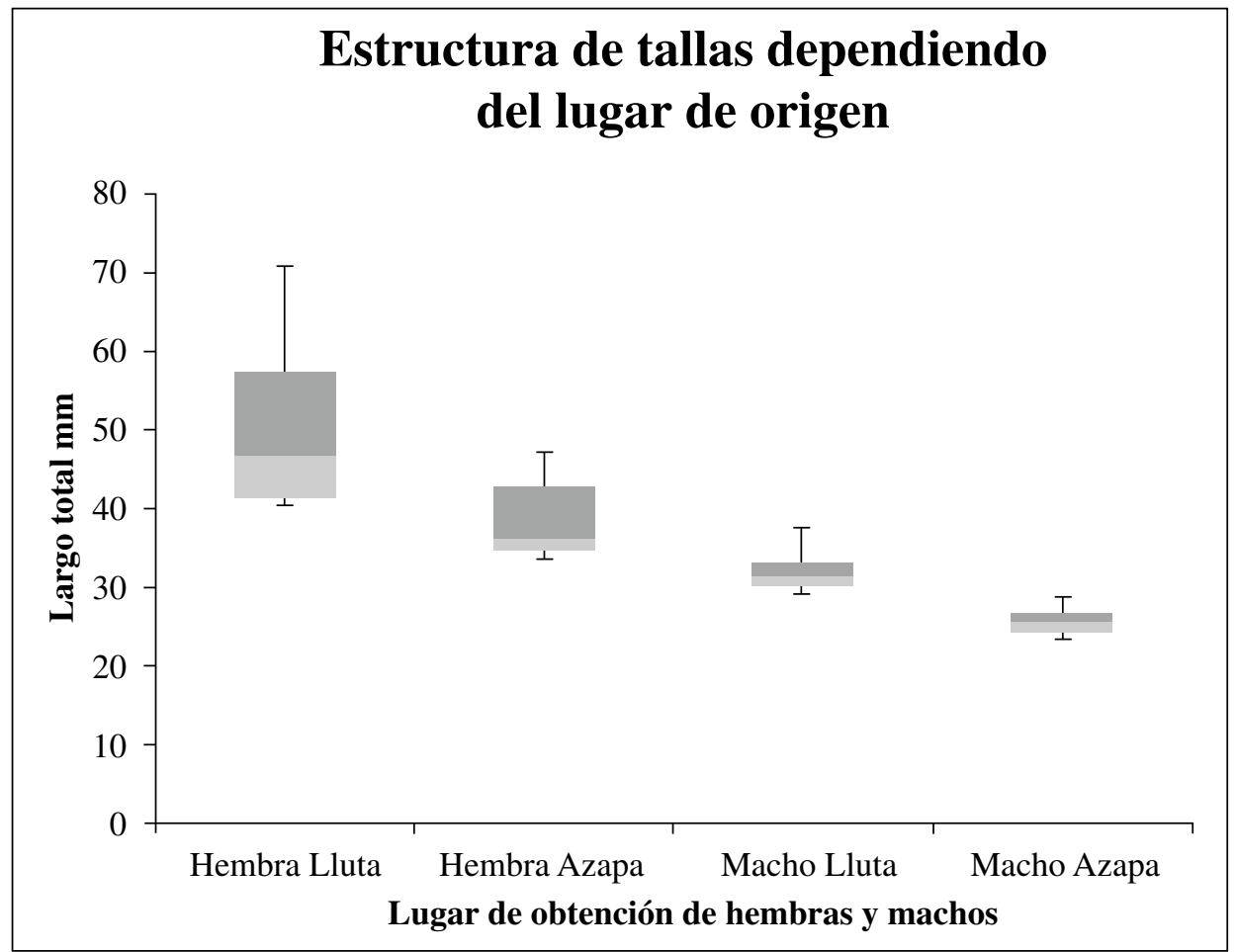

Figura 1. Esquematiza los valores percentiles, mediana y varianza de los tamaños que alcanzaron las Gambusia holbrooki capturadas, se ordenan comparando hembras y machos haciendo la diferencia en el valle del que fueron obtenidos los ejemplares, enero 2013. 
podemos observar que casi todos los valores son cercanos a 1, excepto el caso de los peces machos de Lluta (que obtuvo un $\mathrm{K}=0,87 \pm 0,1$ ).

Estudios que utilizaron el tamaño corporal de Gambusia puncticulata para indicar los efectos de la contaminación humana sobre la biología del pez evidenciaron que las hembras que habitan en ambientes altamente contaminados (columna de agua, presencia de amonio, nitrito, nitrato y coliformes fecales; sedimento cobre, plomo y zinc) alcanzan mayor tamaño, sus resultados lo justifican porque la contaminación antrópica genera un enriquecimiento de nutrientes (sustancias nitrogenadas), lo que conlleva un aumento en la proliferación de productores primarios aumentando así la disponibilidad de alimento para G. puncticulata (Cabrera et al., 2008). A pesar que se puede hacer una analogía con nuestros resultados, es poco probable que la disponibilidad de alimento, producto del enriquecimiento del agua con nitratos, sea el causante de la diferencia en el tamaño, debido a que en las aguas que habitan los peces no fue detectada la presencia de nitratos (Tabla 2), por otro lado, Lluta como Azapa suponen la misma condición de manejo para evitar acumulación de material que a la postre originan la proliferación de plantas y algas acuáticas. Por lo que la explicación pudiera relacionarse con la calidad del agua y la proporción de los minerales disueltos, en vez de contaminación antrópica.

\section{Determinación de presencia y concentración de boro en las aguas y en el tejido de Gambusia holbrooki}

Los análisis hechos a la calidad de agua demostraron una diferencia sustancial entre los dos ríos, prácticamente todos los parámetros fueron doblados o triplicados (Tabla 2). Cabe destacar la concentración de boro disuelto en las aguas de ambos ríos, las aguas del río Lluta tienen una magnitud 10 veces mayor que las aguas del río San José. El único valor en común fue el $\mathrm{pH}$, dado por el origen altiplánico de ambos ríos, y la diferencia en la conductividad seguramente se debió a los valores de los iones de cloruros y sodio, que en las aguas del río Lluta presentan los mayores valores. Los parámetros que se obtuvieron para caracterizar la calidad del agua de los ríos Lluta y San José en el presente estudio concuerdan con los valores históricos informados por Torres y Acevedo (2008), Albornoz et al. (2007), Campos et al. (2007).
Tabla 2. Presenta las concentraciones de elementos químicos y parámetros químico-físicos obtenidos de los estanques de regadíos que se encuentran en las parcelas del valle de Lluta y Azapa, utilizando espectrofotometría. Enero 2013.

\begin{tabular}{lccc}
\hline \multicolumn{1}{c}{ Análisis } & & Parcela de Lluta & Parcela de Azapa \\
\hline Boro & $\mathrm{mg} / \mathrm{l}$ & 12,16 & 1,26 \\
Conductividad & $\mathrm{S} / \mathrm{m}$ & 3,11 & 0,77 \\
$\mathrm{pH}$ & & 8,23 & 8,74 \\
Cloruros & $\mathrm{mg} / \mathrm{l}$ & 660,3 & 250 \\
Calcio & $\mathrm{mg} / \mathrm{l}$ & 280,23 & 110 \\
Magnesio & $\mathrm{mg} / 1$ & 36,2 & 17,24 \\
Sodio & $\mathrm{mg} / 1$ & 308,3 & 98 \\
Potasio & $\mathrm{mg} / 1$ & 35,2 & 9 \\
Sulfato & $\mathrm{mg} / 1$ & 410 & 180 \\
Nitratos & $\mathrm{mg} / 1$ & No detectado & No detectado \\
\hline
\end{tabular}

La cuantificación de boro en los tejidos de Gambusia holbrooki indica el siguiente contraste, más contenido de boro en los peces lluteños y menor concentración de boro en los peces azapeños (Figura 2), si se observa en detalle: la concentración de boro presente en el estanque de Azapa es de 1,26 $\mathrm{mg}$ de boro/l, mientras que el boro presente en los tejidos de los peces azapeños es cinco veces mayor $(5,48 \mathrm{mg}$ de boro $/ \mathrm{kg})$. En tanto, la concentración de boro que hay en las aguas del río Lluta es de 12,16 mg de boro/l, y en vez de aumentar la concentración de boro en los tejidos del pez, como fue el caso anterior, disminuyó a 7,89 $\mathrm{mg}$ de boro $/ \mathrm{kg}$. Sin embargo, estadísticamente no hay diferencias significativas entre las dos poblaciones $(\mathrm{P}=0,07)$.

El boro es un elemento bioactivo necesario en la dieta, por ejemplo el pez cebra requiere de boro para completar su ciclo de vida, además se demostró en ratas que pequeñas concentraciones de boro son necesarias para la formación de trabéculas óseas (Nielsen, 2008). No obstante, se ha determinado que niveles altos de boro (ácido bórico principalmente) son tóxicos en ambientes acuáticos, niveles sobre $0,34 \mathrm{mg} / \mathrm{L}$ de boro comienzan a causar daños a los organismos acuáticos (Schoderboek et al., 2011) y si el boro se combina con la sal ocurre sinergia en los efectos, por ejemplo en el camarón de río Litopenaeus vannemei (Li et al., 2008) se identificó que concentraciones altas de boro y un aumento en la salinidad incrementa la tasa de mortalidad. Por otro lado se informó una estrategia de los peces Paralichthys olivaceus y Pagrus major para resistir los efectos deletéreos del boro: el pez aumenta su tamaño corporal, es decir, mientras más grande 


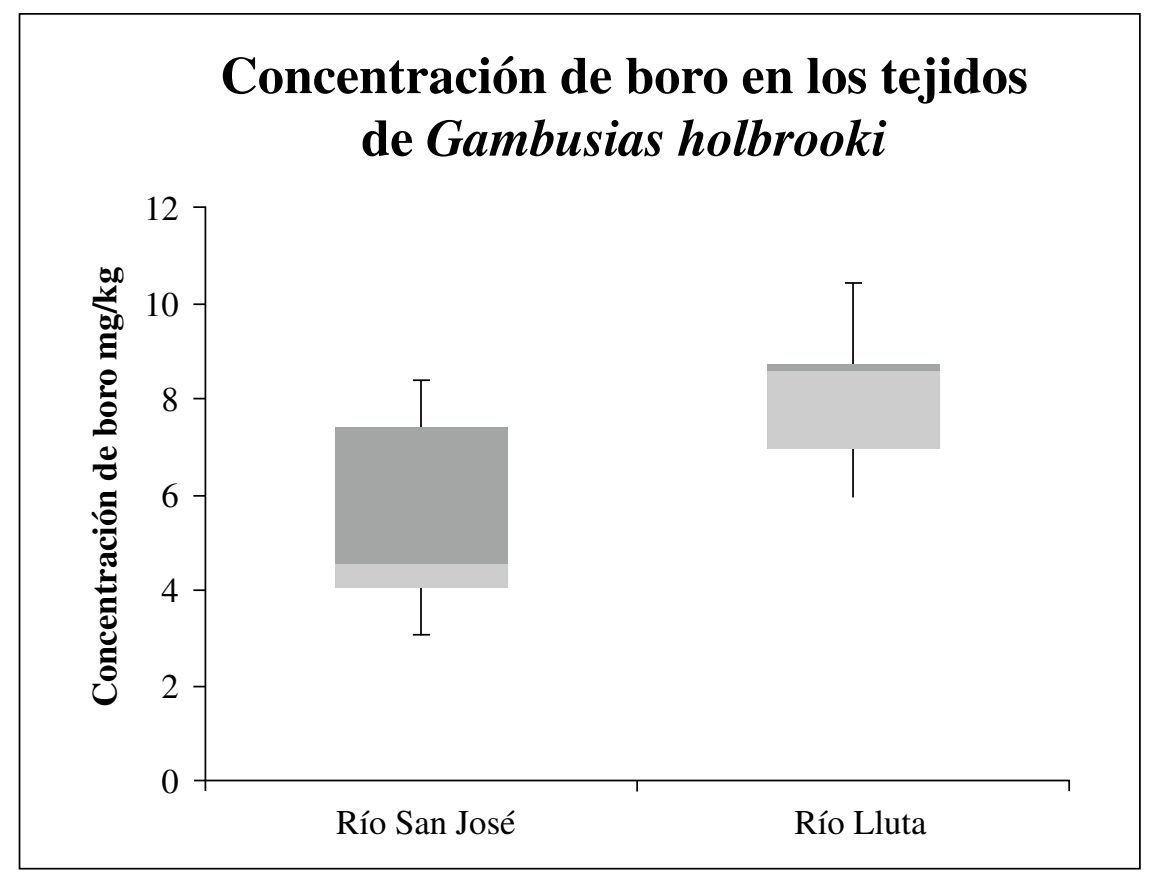

Figura 2. Representa las concentraciones de boro obtenidas en los tejidos de Gambusia holbrooki en relación al hábitat de origen, río San José (valle de Azapa) y río Lluta (valle de Lluta); se indica la mediana y la varianza asociada a cada valor. Los datos fueron obtenidos mediante espectrofotometría en enero de 2013.

sea el pez más tiempo resistirá los niveles letales de boro (Furuta et al., 2007).

En nuestro estudio los niveles de boro que presentan las aguas del río Lluta es el más alto $(12,16 \mathrm{mg} / \mathrm{l})$ y la población que ahí habita alcanzó el mayor tamaño, aparentemente la interacción con cloruros, sodio y sulfatos no afectaron negativamente al tamaño corporal de la población lluteña. Sin embargo, se necesita de bioensayos toxicológicos para determinar el rol del boro en la biología de G. holbrooki.

\section{Conclusiones}

Se determinó que la presencia y concentración de boro difiere en ambos ríos, siendo el río de Lluta el que presenta mayor cantidad de boro.
Estadísticamente no existen diferencias significativas en el contenido de boro en los tejidos de Gambusia holbrooki de las poblaciones lluteñas y azapeñas $(\mathrm{P}=0,07)$.

Los resultados de la caracterización morfológica de los ejemplares capturados evidenció que los peces de Lluta son más grandes y más pesados que los peces oriundos del valle de Azapa.

\section{Agradecimientos}

Agradezco a la Dra. Elizabeth Bastías que me permitió trabajar en las dependencias de la parcela de Lluta, a Juana Morales que hizo el contacto para trabajar en una parcela del valle de Azapa, a todas las personas que nos apoyaron directa e indirectamente en esta investigación. 


\section{Literatura Citada}

Albornoz, F.A.; Torres, M.; Tapia, A.; Acevedo, E. 2007. Cultivo de tomate (Lycopersicon esculentum Mill.) hidropónico con agua desalinizada y desborificada en el valle de Lluta. Idesia, 25(2): 73-80.

Cabrera, Y.; Aguilar, C.; González-Sansón, G.

2008. Indicadores morfológicos y reproductivos del pez Gambusia puncticulata (Poeciliidae) en sitios muy contaminados del río Almendares, Cuba. Revista de Biología Tropical, 56(4): 1991-2004.

CADE-IDEP consultores en ingeniería

2004. Diagnóstico y clasificación de los cursos y cuerpos de agua según objetivos de calidad: Cuenca del Río Lluta. Dirección General de Aguas-Ministerio de Obras PúblicasGobierno de Chile 105 p.

Campos, H.; Díaz, G.; Campos, C.

2007. Aportes sedimentarios de los ríos Lluta y San José en la zona costera de la rada de Arica, Chile. Idesia, 25(2): 37-48.

Cifuentes, R.; González, J.; Montoya, G.; Jara, A.; Ortiz, N.;

Piedra, P.; Habit, E.

2012. Relación longitud-peso y factor de condición de los peces nativos del río San Pedro (cuenca del río Valdivia, Chile). Gayana, 75(2): 101-110.

Figueroa, L; Tapia, L.; Bastías, E., Escobar, H.; Torres, A. 1994. Niveles de boro en aguas de riego y suelos que sustentan olivos en el Norte de Chile. Idesia, 13: 63-68.

Furuta, T.; Iwata, N.; Kikuchi, K.

2007. Effects of fish size and water temperature on the acute toxicity of boron to japonese flounder Paralichthys olivaceus and red sea bream Pagrus major. Fisheries Science, 73: 356-363.

Granado, C.

2002. Ecología de Peces. Ediciones Universidad de Sevillasecretariado de Publicaciones, Sevilla-España, 361 p.

Li, E.; Xiong, Z.; Chen, L.; Zeng, C.; Li, K.

2008. Acutre toxicity of boron to juvenile white shrimp, Litopenaeus vannamei, at two salinities. Aquaculture, 278: 175-178.

Neghme, A.; Gutiérrez, J.; Albi, H.

1947. Control de anofelismo en las zonas maláricas chilenas. Boletín de la Oficina Sanitaria Panamericana. 157-158.
Nielsen, F.

2008. Is boron nutritionally relevant? Nutrition Reviews, 66(44): 183-191.

Oriol-Vidal, N.; Araguas, R.; Fernández-Cebrian, R.; Diez, D.; García, J.

2012, SPN diversity in introduced populations of the invasive Gambusia holbrooki. Ecology of Freshwater Fish, 21: 100-108.

Osorio, M.; Mesías, J.M.; Araya, R.

2013. Estiércol de vacuno estabilizado y daño por boro en lechuga (Lactuca sativa L.) cv. del río, en el valle de Lluta, Chile. Idesia, 31(1) 15-25.

PUC-Pontificia Universidad Católica

2009. Estudio de Calidad de Aguas del Río Lluta. Dirección General de Aguas - Ministerio de Obras Públicas - República de Chile, 47 p.

Pyke, G.

2005. A review of the biology of Gambusia affinis and $G$. holbrooki. Reviews in Fish Biology and Fisheries, 15: 339-365.

Ruiz-Navarro, A.; Moreno-Valcárcel, R.; Torralva, M.; Oliva-

Paterna, F.

2011. Life-history traits of the invasive fish Gambusia holbrooki in saline streams (SE Iberian Peninsula): Does salinity limit its invasive success?. Aquatic Biology, 13: 149-161.

Schoderboeck, L.; Mühlegger, S.; Losert, A.; Gausterer, C.;

Hornek, R.

2011. Effects assessment: boron compounds in the aquatic environment. Chemosphere, 82: 483-487.

Torres, A.; Acevedo, E.

2008. El problema de salinidad en los recursos suelo y agua que afectan el riego y cultivo en los valles de Lluta y Azapa en el norte de Chile. Idesia, 26(3): 31-44.

UTA-Universidad de Tarapacá

1997. Estudio de Impacto Ambiental Proyecto: Ampliación de la capacidad de producción de agua potable en Arica; captaciones costeras, sondaje Lluta bajo y planta desalinizadora. Empresa de Servicios Sanitarios Tarapacá S.A.-ESSAT, 685 p. 